\title{
Measure of Singular Value Decomposition (M- SVD) based Quality Assessment for Medical Images with Degradation
}

\author{
Ersin Elbasi \\ College of Engineering and Technology, American University of the Middle East, Kuwait \\ ersin.elbasi@aum.edu.kw
}

\begin{abstract}
We use images in several important areas such as military, health, security, and science. Images can be distorted during the capturing, recording, processing, and storing. Image quality metrics are the techniques to measure the quality and quality accuracy level of the images and videos. Most of the quality measurement algorithms does not affect by small distortions in the image. Magnetic Resonance Imaging (MRI), Computed Tomography (CT), and Ultrasonic Imaging (UI) are widely used in the health sector. Because of several reasons it might be artifacts in the medical images. Doctor decisions might be affected by these image artifacts. Image quality measurement is an important and challenging area to work on. There are several metrics that have been done in the literature such as mean square error, peak signal-noise ratio, gradient similarity measure, structural similarity index, and universal image quality. Patient information can be an embedded corner of the medical image as a watermark. Watermark can be considered one of the image distortions types. The most common objective evaluation algorithms are simple pixel based which are very unreliable, resulting in poor correlation with the human visual system. In this work, we proposed a new image quality metric which is a Measure of Singular Value Decomposition (M-SVD). Experimental results show that novel M-SVD algorithm gives very promising results against Peak Signal to Noise Ratio (PSNR), the Mean Square Error (MSE), Structural Similarity Index Measures (SSIM), and 3.4. Universal Image Quality (UIQ) assessments in watermarked and distorted images such as histogram equalization, JPEG compression, Gamma Correction, Gaussian Noise, Image Denoising, and Contrast Change.
\end{abstract}

Keywords: Image quality measurement, $M-S V D$, image distortion, watermarking, medical image.

Received September 7, 2020; accepted February 1, 2021

https://doi.org/10.34028/iajit/18/5/14

\section{Introduction}

Multimedia elements such as image and video have been getting importance with information technology. It can be distributed easily via internet technology. Content owners concern about security issues and possible modification on multimedia elements by third parties using new technology tools. Image quality is also very important especially some sensitive application areas such as military, health, science, and security. Images can be distorted during the capturing, recording, processing, and storing. Image quality metrics are the techniques to measure the quality and quality accuracy level of the images and videos. There are two types of measurement which are subjective measurement and objective measurement [13]. In subjective measurement, experts selected from pool check images and give a quality score for each image. Mean Opinion Score (MOS) is calculated from expert scores. Subjective measurement is based on the human perception system. It is more expensive than an objective measurement. Because in objective measurement we have computing algorithms and measure automatically derived from this algorithm. Subjective measurement can be also used to verify new image quality measurement metrics. There are two types of subjective measurement based on given sources. In a single stimulus, expert can see only the test image without seeing original image. In double stimulus, experts can see both source and test images, and they can compare this to images. There is a possibility that an expert can be affected by environmental conditions and viewing points [20, 24].

There are several objective measurement metrics such as mean square error, peak signal to noise ratio, structural similarity index, gradient similarity index, mean square error, and universal image quality [23]. We can classify objective measurement based on the availability of source image as follows:

- No reference quality measurement

- Full reference quality measurement

- Reduced reference quality measurement

Both test image and source image are available in full reference, only test image available in no reference, extracted features are used in reduced reference-quality measurements. No reference image quality measurement is more complicated than the other two methods. There are several image quality 
measurements such as distortion, noise, contrast, sharpness, colour, dynamic range, lens, and artifacts [3, 14, 15]. Medical imaging is one of the important application areas of image processing such as Magnetic Resonance Imaging (MRI), Computed Tomography (CT), and Ultrasonic Imaging (UI). Nowadays doctors get the benefit of medical imaging technology for diagnosis and patient treatment. In addition to this clinics process, print, transfer, and store images. All these processes can affect medical image quality $[5,16]$. Usually, we do not want any small distortions in medical images. Because decisions can be very critical from medical images, even small pixel changes can change the decision in the wrong way.

Watermarking is one of the multimedia security techniques. A logo, stamp, random number, or image can be hidden into the cover image for copyright protection. Patient information can be an embedded corner of the medical image as a watermark [1, 4]. Watermark can be considered one of the image distortions types. In this work, we proposed a new image quality metric which is Measure of Singular Value Decomposition (M-SVD). M-SVD shows promising results in watermarked medical images compared to other common quality assessments. We can classify watermarking via several categories:

- Non-blind, semi-blind and blind watermarking.

- Visual and transparent watermarking.

- Image/logo/stamp and Pseudo Random Number (PRN) watermarking.

- Spatial domain and transformation-based watermarking.

In spatial domain watermarking, the watermark is embedded directly into the cover image by changing the image pixel values. On the other hand, images can be transferred into another domain such as discrete wavelet, Fourier, discrete cosine, or radon transformation. Both methods have some advantages and disadvantages. There are several image processing attacks into watermarked images such as adding noise, histogram equalization, rotation, resizing, rewatermarking, contrast adjustment, and cropping. Some of the developed watermarking algorithms are successful against a group of attacks, and not resist against another group of attacks $[6,19]$.

The most common objective evaluation algorithms are simple pixel based such as the Mean Square Error (MSE), is very unreliable, resulting in poor correlation with the human visual system. In this work, we proposed a novel image quality measurement metric which is based on Singular Value Decomposition, MSVD, for gray-scale and color images that can express the quality of distorted images numerically or graphically.

This paper is organized as follows. A brief introduction to related works is presented in section 2 . Section 3 describes the current and proposed image quality measurement techniques. Experimental results are represented in section 4 , and conclusions in section 5.

\section{Literature Review}

Contrast enhancement is one of the most frequently used methods to enhance natural images. Gu et al. [13] proposed a new reduced reference image quality metric for images which has contrast changed. Large contrast changed images database used in this work for both subjective and objective measurements. Experimental results show that the proposed novel reduced reference image quality metric gives better results than other common measurements. We use images in several important areas such as military, health, security, and science. The quality of the image is very important to extract high-quality feature vector from images and videos. Panetta et al. [20] developed a new transform domain quality measurement algorithm that has no reference and no parameter.

Most of the quality measurement algorithms does not affect by small distortions in the image. Especially medical images we are using in the health such as MR, MRI and ultrasound. Quality measurement must be very sensitive. The proposed algorithm automatically selects parameters and gives very powerful results [20]. Wang et al. [24] proposed objective waveletbased image quality measurement for the Region Of Interest (ROI). In some of the image and video applications ROI is very important for the Human Vision System (HVS). In this method, the authors applied the discrete wavelet transformation to the coded selected area of the image to measure the quality of the multimedia elements. This method gives very promising results especially for compressed images.

Face recognition is one of the important image processing application. Especially face area quality in the image is critical to recognize faces correctly. There are several supervised solutions for face recognition and quality measurement of the faces in the literature. Terhorst et al. [23] proposed a new unsupervised algorithm. Quality labels are not used in the given algorithm. An unsupervised face image quality estimation algorithm has been compared with the 6 most common supervised image quality measurements. Experimental results show that unsupervised estimation gives very good results in face images. This method can be easily integrated to face recognition applications. During the image acquisition images can be out of focus, it might be some blurring. It makes image and feature vector quality very low. Most of the image quality metrics do not count out of focus blurring in the images.

Liu et al. [15] developed a new algorithm that is strong for quality measurement of out of focus blurring images. There are 150 out of focus images used in the experiments. Proposed objective quality measurement 
is compared with other algorithms and results are verified with subjective measurement. MRI is widely used in the health sector. Because of several reasons, it might be artifacts in the image. Doctor decisions might be affected by these image artifacts. There are some works in the literature for MRI enhancement using deep learning algorithms. In this works, MRI images directly used in the training. Some of the images have artifacts and noises, it affects the results and enhancement quality. Jeelani et al. [14] proposed a new methodology in MRI deep learning training for image enhancement. In this method, a loss function finds out artifacts and noisy MRI images and does not use them in the training process. Results show that the proposed algorithm gives better results than noisy training algorithms.

Bognar [3] developed a new algorithm based on SKFCM image segmentation and Structural Similarity Index Measures (SSIM) image quality measurement. No reference image quality method measures the quality of the human lung CT images. It can be used for the quality of the scanned CT and enhancement quality of the medical image. During the experiments, several common image processing attacks are applied to the CTs. Results show that the proposed algorithm is very effective for human lung CT images. Peak Signal to Noise Ratio (PSNR), SSIM, and Root Mean Square Error (RMSE) are the most widely used objective image quality metrics. Mason et al. [16] has compared objective and subjective measurement qualities using MR images. Total 1017 MR images used, 414 of them are degraded images. Performance is evaluated using the Spearman rank-order correlation coefficient. Based on the experimental results, RMSE and SSIM give better Spearman's Rank Correlation Coefficient (SROCC) value than other 6 image quality measurement methods. This result should be considered in medical image quality measurement.

Blurring, compression, and filtering are the most annoying distortion types in images. There are several quality metrics are used to find out distortion and measure the quality of the images. Chetouani [5] proposed a blind fusion-based stereoscopic image quality metric for blur distortion. Features are extracted from stereoscopic images, then support vector machine is used to combine all these features. The proposed method gives very good results in both grey and colour images. We need a high resolution for some of the images. It is a very difficult problem to measure the quality of the interpolated images. Chen et al. [4] proposed a new algorithm to handle this problem. In this method hybrid, no reference, and reduced reference-based quality measurement used. The proposed method compared with several quality metrics and verified by subjective measurement. Results show that the developed hybrid method gives better result than regular quality metrics.

Image quality measurement is an important and challenging area to work on. There are several metrics that have been done in the literature. AlZahir and Kashanchi [1] proposed the statistical function Gaussian copula to find out the quality of the images if the data distribution is unknown. This function provides image distortion or tamper information. Results are compared with three quality measurement methods which are universal quality measure, structural similarity, and visual information fidelity. Experimental results show that the proposed function gives the same results with $99 \%$ accuracy with the common three algorithms. Medical images such as MR, Ultrasound, and CT most commonly analysed by radiologists. There are several reasons not to analyse fully automatic. One of the reasons is the low image quality gives wrong information to the doctors. Outtas et al. [19] worked on no-reference quality metrics for ultrasound human liver images. Scores are obtained from radiologists using subjective quality measurement. There are several NR quality measurements tested and four of them gave very successful results when verified with subjective measurement.

Min and Park [17] proposed image quality measurement for a mobile display of outdoor images. Blurring, sharpening, noise, colourfulness, and lightness are the most common factors in outdoor image qualities. Contrast and colourfulness are selected for two important features for image quality metrics. Based on this feature RMSE and SSIM give very promising results in the mobile display of the outdoor images. There are several image quality metrics for distorted images. It is difficult to use most of them in optimization problems in image applications because of mathematical properties. Bae and Kim [2] proposed a new algorithm which is DCT based quality metric for optimization problems. The weighted mean is used after converting the image into the discrete cosine transformation. The proposed algorithm gives very promising results after common attacks such as noise, filtering, histogram equalization, and sharpening.

Watermarking is one of the distortion types for the images. If the embedding algorithm is very powerful and there are no attacks to the image after watermarking, it will be very small distortion that happens to the image. Nezhadarya et al. [18] proposed a quality monitoring algorithm using spread spectrum watermarking. It gives very promising results in the quality measurement if compared to PSNR values. Quality measurement is one of the performance measurements of the watermarking algorithms. The watermarked multimedia element can be distorted after embedding and possible attacks. PSNR values and extraction results give information about watermarking performance. Taha and Taha [22] proposed a new quality metric for watermarked high and low textured images. Texture mapping is used in quality metrics. 
Experimental results show that the proposed algorithm gives better results than frequently used 3.1. PSNR and SSIM metrics.

\section{Quality Measurement and Proposed Assessment}

\subsection{Peak Signal to Noise Ratio (PSNR)}

PSNR is one of the image quality measurement metrics. It represents a ratio between the highest value of a signal and the power of distorted noise. There are several metrics in image quality, and it is difficult to decide which one is better because of the different perspective [7, 21].

Let's say we have the original image and possibly a distorted image which is two-dimensional array $A$. We can represent the PSNR value as follows:

$$
\begin{gathered}
\text { PSNR }=20 x \log _{10} \frac{\text { Maximum Image } A}{\sqrt{\text { Mean Square Error }}} \\
\text { Mean Square Error }=\frac{1}{a x b} \sum_{0}^{a-1} \sum_{0}^{b-1}\|M(a, b)-N(a, b)\|^{2}
\end{gathered}
$$

Where $A$ is the image matrix, $a$ is the number rows and $b$ is the number columns in the image. $M$ is the distorted image and $N$ is the reference image. MSE measures differences between distorted and reference images. If the PSNR value is high, we have less or no distortion, otherwise, we have more distortion.

\subsection{Structural Similarity Index (SSIM)}

The structural similarity index measures the differences between two images. One of the images is the original, not distorted image [21].

$$
\begin{gathered}
S S I M=\left[\frac{2 a_{1} a_{2}}{a_{1}^{2} a_{2}^{2}}\right] \times\left[\frac{2 b_{1} b_{2}}{b_{1}^{2}+b_{2}^{2}}\right] \times\left[\frac{b_{12}}{b_{1} b_{2}}\right] \\
S S I M=\left[\frac{2 a_{1} a_{2}}{a_{1}^{2}+a_{2}^{2}}\right] \times\left[\frac{2 b_{12}}{b_{1}^{2}+b_{2}^{2}}\right]
\end{gathered}
$$

Where $a$ is the mean and $b$ is the variance of images. SSIM is the product of local luminance and local covariance.

Structural similarity (DSSIM) is another quality measurement that is extracted from the structural similarity index metric. There are three major features are used in DSSIM that are correlation, contrast, and luminance.

$$
\begin{aligned}
& \qquad \operatorname{SSIM}=[\operatorname{Luminace}(a, b)]^{i} x \\
& =[\text { Contrast }(a, b)]^{j} x= \\
& {[\text { Correlation }(a, b)]^{z} \text { Structural Similarity }=\frac{1-\operatorname{SSIM}(a, b)}{2}}
\end{aligned}
$$

\subsection{Mean Square Error (MSE)}

MSE find the summation of the differences between the original image and the distorted image. If the MSE value is closer to zero, it is better. Each pixel of an image has a greyscale value. When there is a distortion or changes happens, the pixel number changes. MSE measures how much changes from the reference image [8].

Let's say we have two image matrix $m(a, b)$ and $n$ $(a, b)$ where $\mathrm{m}$ is the full reference image and $n$ is the distorted image.

$$
\text { Mean Square Error }=\frac{1}{A x B} \sum_{a=0}^{A} \sum_{b=0}^{B}\left[n(a, b)-m(a, b]^{2}\right.
$$

We can say that the MSE value is the absolute error between the two image matrices. RMSE is another type of quality measure which is calculated from MSE.

$$
\text { Root MSE }(\alpha)=\sqrt{\text { Mean Square Error }(\alpha)}
$$

Where $\alpha$ estimate parameter set for reference and distorted images.

\subsection{Universal Image Quality (UIQ)}

MSE and SSIM quality measurement metrics calculate the summation of distortion error between two images. UIQ is a new model that calculates total distortion using luminance distortion, contrast distortion, and loss of correlation [8].

$$
\begin{gathered}
\text { Universal Image Quality }=\frac{4 x S t_{a b} x M_{x} x M_{b}}{\left(S t_{b}^{2}+S t_{a}^{2}\right) x\left(M_{x}^{2}+M_{b}^{2}\right)} \\
M_{x}=\frac{1}{N} \sum_{i=1}^{N} x_{i} \\
M_{b}=\frac{1}{N} \sum_{i=1}^{N} b_{i} \\
S t_{b}^{2}=\frac{1}{N-1} \sum_{i=1}^{N}\left(x_{i}-M_{x}\right)^{2} \\
S t_{b}^{2}=\frac{1}{N-1} \sum_{i=1}^{N}\left(b_{i}-M_{b}\right)^{2}
\end{gathered}
$$

Where $N$ is the number of pixels in the image.

\subsection{Gradient Similarity Measure (GSM)}

Gradient similarity measure changes between two images in contrast and image structure. Measuring both changes make quality metric more efficient. The gradient similarity measure is more robust, effective, and faster than other common image quality metrics.

$$
\text { Gradient Similarity }=\frac{2 x G r_{x} x G r_{y}+\alpha}{G r_{x}^{2}+G r_{y}^{2}+\alpha}
$$

Where $G r$ is the gradient value and $\alpha$ is the constant value.

\subsection{Measure of Singular Value Decomposition (M-SVD)}

The measure of singular value decomposition is a novel measurement metric. The image can be represented as matrix $\mathrm{B}$ which is the product of three matrices orthogonal $\mathrm{U}$ and $\mathrm{V}$, the singular values of $\mathrm{B}$ is $\mathrm{S}$.

$\mathrm{U}$ : Left singular values of $\mathrm{B}$

$\mathrm{V}$ : Right singular values of $\mathrm{B}$

The proposed algorithm calculates distances between 
singular values between the original image and possibly distorted image.

$$
\text { Distance between singular values }\left(D_{a}\right)=\sqrt{\sum_{a=1}^{M}\left(F_{a}-F_{a}^{\prime}\right)^{2}}
$$

$F_{\mathrm{a}}$ and $F_{\mathrm{a}}{ }^{\prime}$ are singular values of original and distorted images. If $b$ is the block size of the image and $M$ is the image size, there are $\left(\frac{M}{b} x \frac{M}{b}\right)$ blocks in the image.

Measure of Singular Value Decomposition $=\frac{\sum_{a=1}^{\left(\frac{M}{b} \times \frac{M}{b}\right)}\left|D_{a}-D_{m}\right|}{\left(\frac{M}{b} \times \frac{M}{b}\right)}$

Where $D_{m}$ is the midpoint of the values when we order them.

\section{Experimental Results}

Discrete wavelet domain-based watermarking gives very promising results for medical image watermarking. In this method, the watermark is embedded into the high frequencies which are edges of the image. Some of the attacks in watermarking directly destroy the image, some of them let the watermark algorithm does not work properly. In Table 1 several distortion methods applied into the watermarked medical images such as histogram equalization, gamma correction, JPEG compression, Gaussian Noise, Gaussian Blur, Image Denoising, and Contrast change. Image quality measurement algorithms have different measurement scales for example PSNR value 40, and more means two images are almost the same. In the Table 1, we converted each method measurement results between 0 to 1. SSIM quality measurement gives better results in histogram equalization and Gaussian noise. Novel proposed algorithm M-SVD gives more accurate results than 5 other distortions $[9,10]$.

Table 1. PSNR, MSE, SSIM, UIQ and M-SVD image quality measurement after distorted watermarked image.

\begin{tabular}{|c|c|c|c|c|c|}
\hline Distortion Type & PSNR & MSE & SSIM & UIQ & M-SVD \\
\hline Histogram Equalization & 0.891 & 0.875 & 0.952 & 0.911 & 0.925 \\
\hline Gamma Correction & 0.875 & 0.851 & 0.924 & 0.923 & 0.962 \\
\hline JPEG Compression & 0.901 & 0.852 & 0.907 & 0.899 & 0.915 \\
\hline Gaussian Noise & 0.925 & 0.903 & 0.934 & 0.852 & 0.906 \\
\hline Gaussian Blur & 0.887 & 0.921 & 0.897 & 0.752 & 0.917 \\
\hline Image Denoising & 0.906 & 0.812 & 0.902 & 0.792 & 0.926 \\
\hline Contrast Change & 0.912 & 0.792 & 0.884 & 0.905 & 0.947 \\
\hline
\end{tabular}

Table 2 shows quality measurement results in medical images for level 1, level 2, level 3, and level 4. In all levels, M-SVD measurement has more accuracy than other measurements. Quality differences between M-SVD and other measurements PSNR, MSE, SSIM, and UIQ are getting more in a high number of levels.

Table 2. PSNR, MSE, SSIM, UIQ and M-SVD image quality measurement with 4 level distortion.

\begin{tabular}{|c|c|c|c|c|c|}
\hline Distortion Level & PSNR & MSE & SSIM & UIQ & M-SVD \\
\hline Level 1 & 0.951 & 0.972 & 0.967 & 0.941 & 0.982 \\
\hline Level 2 & 0.925 & 0.924 & 0.959 & 0.929 & 0.974 \\
\hline Level 3 & 0.932 & 0.912 & 0.934 & 0.898 & 0.949 \\
\hline Level 4 & 0.904 & 0.872 & 0.932 & 0.871 & 0.937 \\
\hline
\end{tabular}

In medical imaging, every pixel of the image quality is very important. Because distortion only in a small portion of the image might affect the medical specialist decision. Usually, a watermark is embedded into any corner of the medical image to protect patient medical information. In Figure 1, the mostly used original medical images and watermarked images have been given. Binary watermark is embedded into the high levels of frequencies in LH, HL, and HH bands. PSNR values show that watermarking images do not have any distortion and similarity between the two images [11].

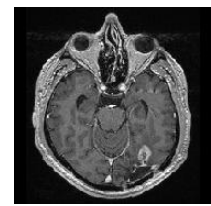

a) Reference MRI.

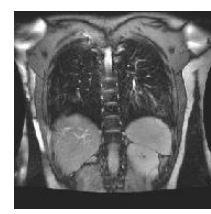

c) Reference body.

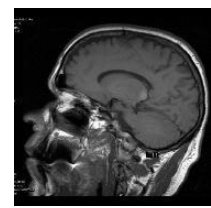

e) Reference brain.

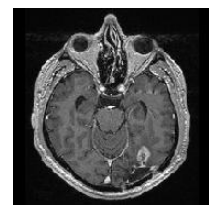

b)Watermarked MRI (PSNR=41.592)

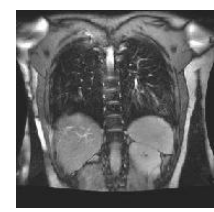
(PSNR=43.183).

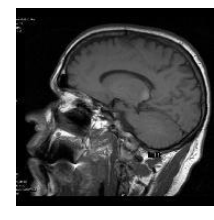

f) Watermarked brain (PSNR=40.673) d) Watermarked body

Figure 1. Original and watermarked medical images MRI, body and brain.

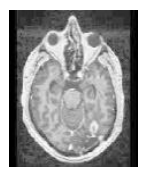

a) Histogram equalization

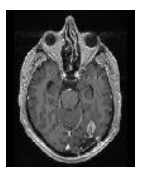

c) JPEG compression

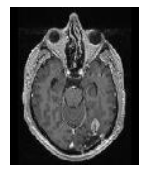

e) Image denoising
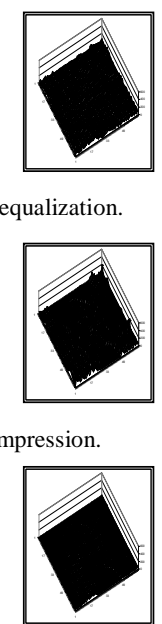

Figure 2. Distortion medical images and maps.
M-SVD image quality metric is used for full color images using a color model which decouples the color and gray-scale information in an image. Our experiments show that using only the luminance component, the measure outperforms UQI, PSNR and MSSIM metrics. When we use the two chrominance layers, the performance of M-SVD becomes slightly higher than other metrics. This shows that M-SVD 
gives similar performance in color images with grayscale image quality measurement.

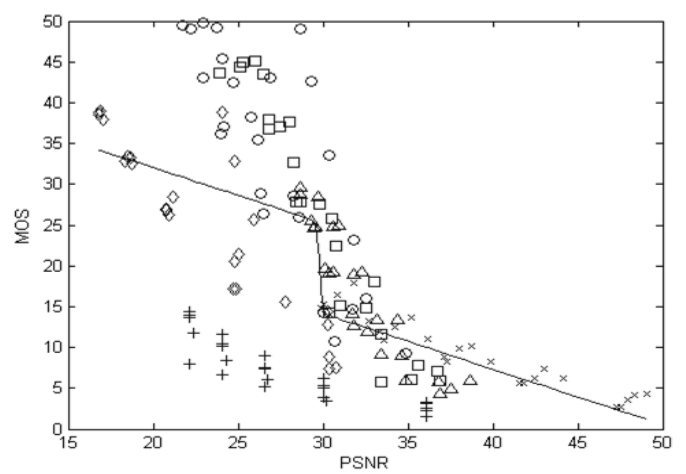

a) PSNR measurement for distortions histogram equalization ( $\square$ ), gamma correction $(\Delta)$, jpeg compression (o), Gaussian noise $(\diamond)$, image denoising $(\mathrm{x})$, contrast change $(+)$

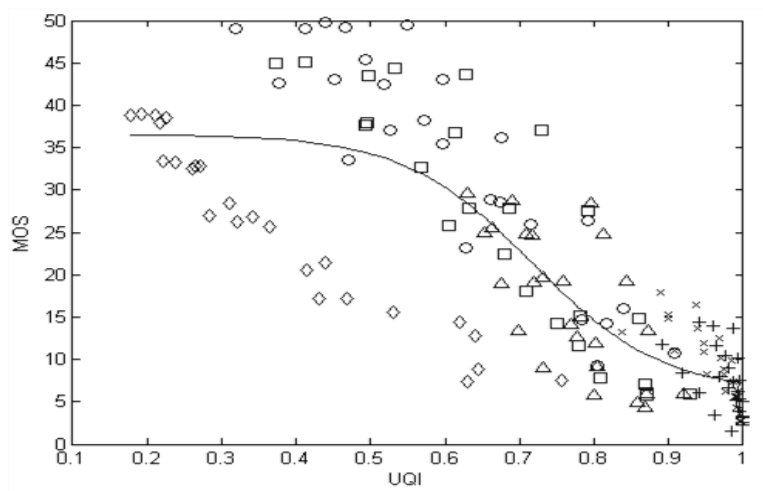

b) UQI measurement for distortions histogram equalization ( $\square)$, gamma correction $(\Delta)$, jpeg compression $(\mathrm{o})$, Gaussian noise $(\diamond)$, image denoising $(\mathrm{x})$, contrast change $(+)$.

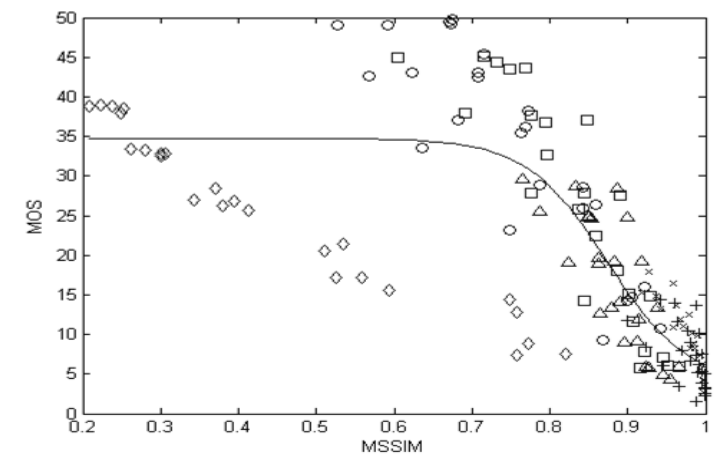

c) MSSIM measurement for distortions histogram equalization ( $\square$ ), gamma correction $(\Delta)$, jpeg compression $(\mathrm{o})$, Gaussian noise $(\diamond)$, image denoising $(\mathrm{x})$, contrast change $(+)$

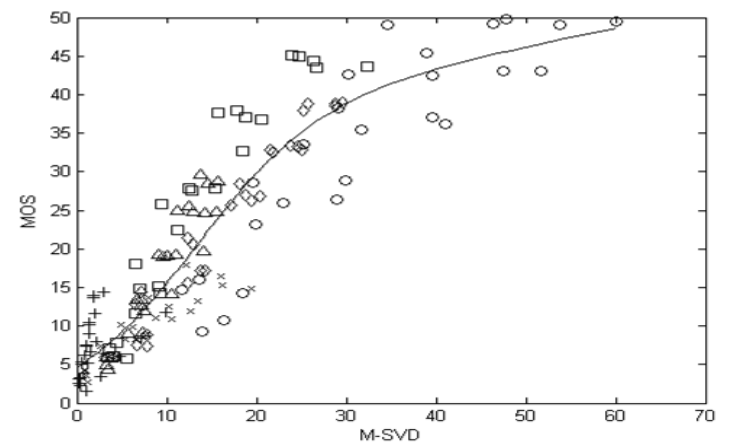

d) M-SVD measurement for distortions histogram equalization ( $\square$ ), gamma correction $(\Delta)$, jpeg compression $(\mathrm{o})$, Gaussian noise $(\diamond)$, image denoising $(\mathrm{x})$, contrast change $(+)$.

Figure 3. Comparison of M-SVD, PSNR, MSSIM and UQI measurement for distortions histogram equalization $(\square)$, gamma correction $(\Delta)$, jpeg compression (o), Gaussian noise $(\diamond)$, image denoising $(\mathrm{x})$, contrast change $(+)$.
Figure 2 shows distorted watermarked medical images and their quality maps. Figure 3 shows a comparison of M-SVD, PSNR, MSSIM, and UQI measurement for distortions histogram equalization (口), gamma correction $(\Delta)$, jpeg compression (o), Gaussian noise $(\diamond)$, image denoising $(\mathrm{x})$, contrast change (+) in watermarked medical images. MOS represents the mean opinion score in subjective measurement. 50 people used in subjective measurement to compare M-SVD, PSNR, MSSIM, and UQI quality measurements. Correlation results show that the proposed M-SVD image assessment gives a very promising result [12].

\section{Conclusions}

Images can be destroyed during capturing, processing, transmission, and storing. Image Quality Measurement is a methodology to find out changes between two images. There are two types of quality measurement assessment: Subjective measurement and objective measurement. Subjective quality measurement is the human perception method based on image view and attributes. Objective quality measurement is a computational method to assess image qualities. In this paper, several objective image quality measurements have been discussed such as mean square error, peak signal-noise ratio, gradient similarity measure, structural similarity index, and universal image quality. There is a novel image quality measurement is proposed in this work which is a M-SVD. The measure of singular value decomposition is a novel measurement metric. The image can be represented as matrix $\mathrm{B}$ which is the product of three matrices orthogonal $\mathrm{U}$ and $\mathrm{V}$, singular values of B is S. Image Quality measurement methods applied several watermarked and possibly attacked medical images such as MRI, CT, and UI. Experimental results show that the novel M-SVD algorithm gives very promising results against PSNR, MSE, SSIM, and UIQ assessments in watermarked and distorted grey or colour images such as histogram equalization, JPEG compression, Gamma Correction, Gaussian Noise, Image Denoising, and Contrast Change. In future research, we will extend the M-SVD-based image quality measure to video sequences.

\section{References}

[1] AlZahir S. and Kashanchi F., "A New Image Quality Measure," in Proceedings of the $26^{\text {th }}$ IEEE Canadian Conference on Electrical and Computer Engineering, Regina, pp. 1-5, 2013.

[2] Bae S. and Kim M., "DCT-QM: A DCT-Based Quality Degradation Metric for Image Quality Optimization Problems," IEEE Transactions on Image Processing, vol. 25, no. 10, pp. 49164930, 2016. 
[3] Bognár G., "Image Quality Measurement for Low-Dose Human Lung CT scans," in Proceedings of the $38^{\text {th }}$ International Conference on Telecommunications and Signal Processing (TSP), Prague, pp. 587-591, 2015.

[4] Chen J., Xu Y., Ma K., Huang H., and Zhao T., "A Hybrid Quality Metric for Non-Integer Image Interpolation," in Proceeding of the $10^{\text {th }}$ International Conference on Quality of Multimedia Experience, Cagliari, pp. 1-3, 2018.

[5] Chetouani A., "A Fusion-Based Blind Image Quality Metric for Blurred Stereoscopic Images," in Proceedings of the International Conference on Advanced Technologies for Signal and Image Processing, Fez, pp. 1-5, 2017.

[6] Elbasi E., "B-DCT based Watermarking Algorithm for Patient Data Protection in IoMT," in Proceedings of the International Conference on Information Security and Cryptology (ISCTURKEY), Ankara, pp. 1-4, 2020.

[7] Elbasi E. and Kaya V., "Robust Medical Image Watermarking Using Frequency Domain and Least Significant Bits Algorithms," in Proceedings of the International Conference on Computing Sciences and Engineering (ICCSE), Kuwait City, 2018, pp. 1-5.

[8] Elbasi E. and Ozdemir S., "Secure Data Aggregation in Wireless Multimedia Sensor Networks via Watermarking," in Proceedings of the $6^{\text {th }}$ International Conference on Application of Information and Communication Technologies, Tbilisi, page 1-6, 2012.

[9] Elbasi E., "M-SVD based Quality Measurement in Hybrid nonblind Watermarked Medical Images", in Proceedings of the IEEE 43rd International Conference on Telecommunications and Signal Processing (TSP), Milano, 2020.

[10] Elbasi E., "Enhancing the Robustness of Watermarked Medical Images Using Heuristic Search Algorithm," in Proceedings of the $7^{\text {th }}$ International Conference on Internet of Things: Systems, Management and Security, Paris, France, pp. 1-5, 2020.

[11] Elbasi E., "Robust Multimedia Watermarking: Hidden Markov Model Approach for Video Sequences," Turkish Journal of Electrical Engineering and Computer Sciences, vol. 18, no. 2, pp. 159-170, 2010.

[12] Ganesan S. and Rabbani M., "Contrast Enhancement using Completely Overlapped Uniformly Decrementing Sub-Block Histogram Equalization for Less Controlled Illumination Variation," The International Arab Journal of Information Technology, vol. 16, no. 3, pp. 389396, 2019.

[13] Gu K., Zhai G., Yang X., Zhang W., and Liu M., "Subjective and Objective Quality Assessment for Images with Contrast Change," in
Proceedings of IEEE International Conference on Image Processing, Melbourne, pp. 383-387, 2013.

[14] Jeelani H., Martin J., Vasquez F., Salerno M., and Weller D., "Image Quality Affects Deep Learning Reconstruction of MRI," in Proceedings of the IEEE $15^{\text {th }}$ International Symposium on Biomedical Imaging, Washington, pp. 357-360, 2018.

[15] Liu Y., Zhai G., Liu X., and Zhao D., "Quality Assessment for Out-of-Focus Blurred Images," Visual Communications and Image Processing (VCIP), Singapore, pp. 1-4, 2015.

[16] Mason A., Rioux J., Clarke S., Costa A., Schmidt M., Keough V., Huynh T., and Beyea S., "Comparison of Objective Image Quality Metrics to Expert Radiologists' Scoring of Diagnostic Quality of MR Images," IEEE Transactions on Medical Imaging, vol. 39, no. 4, pp. 1064-1072, 2020.

[17] Min B. and Park H., "Quantitative Image Quality Evaluation for Mobile Display in Outdoor Environment," in Proceedings of the IEEE $2^{\text {nd }}$ Global Conference on Consumer Electronics, Tokyo, pp. 345-347, 2013.

[18] Nezhadarya E., Wang Z., and Ward R., "Image Quality Monitoring Using Spread Spectrum Watermarking," in Proceedings of the $16^{\text {th }}$ IEEE International Conference on Image Processing, Cairo, pp. 2233-2236, 2009.

[19] Outtas M., Zhang L., Deforges L., Hamidouche W., and Serir A., "Evaluation of No-reference Quality Metrics for Ultrasound Liver Images," in Proceedings of the $10^{\text {th }}$ International Conference on Quality of Multimedia Experience, Cagliari, pp. 1-3, 2018.

[20] Panetta K., Samani A., and Again S., "A Robust No-Reference, No-Parameter, Transform Domain Image Quality Metric for Evaluating the Quality Of Colour Images," IEEE Access, vol. 6, pp. 10979-10985, 2018.

[21] Sever H., Senol A., and Elbasi E., "Block Size Analysis for Discrete Wavelet Watermarking and Embedding A Vector Image As A Watermark," The International Arab Journal of Information Technology, vol. 16, no. 6, pp. 1036-1043, 2019.

[22] Taha D. and Taha T., "Towards a New Metric for Watermarked Image Assessment," in Proceedings of the $2^{\text {nd }}$ International Conference on Electrical, Communication, Computer, Power and Control Engineering, Mosul, pp. 109-113, 2019.

[23] Terhörst P., Kolf J., Damer N., Kirchbuchner F., and Kuijper A., "SER-FIQ: Unsupervised Estimation of Face Image Quality Based on Stochastic Embedding Robustness," in Proceedings of the IEEE/CVF Conference on Computer Vision and Pattern Recognition, 
Seattle, pp. 5650-5659, 2020.

[24] Wang Z., Bovik A., and Lu L., "Wavelet-Based Foveated Image Quality Measurement for Region of Interest Image Coding," in Proceeding of the International Conference on Image Processing, Thessaloniki, pp. 89-92, 2001.

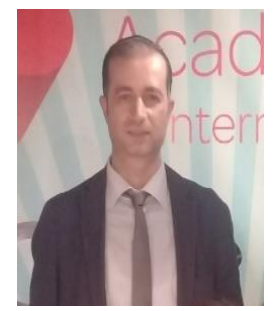

Ersin Elbasi is currently working for American University of the Middle East. He received MSc degree in computer science at Syracuse University; MPhil and $\mathrm{PhD}$ degrees in computer science at Graduate Center, The City University of New York. His research interests include multimedia security, event mining in video sequences and medical image processing. 